\title{
Stabilization of the Precision Positioning Stage Working in the Vacuum Environment by Using the Disturbance Observer
}

\author{
Kazuhiro Moritaa,", Shinji Wakui ${ }^{\mathrm{b}}$ \\ aSemiconductor Process Control Systems Design Division, Hitachi High-Technologies Corporation, 882 Ichige, \\ Hitachinaka-shi, Ibaraki, 312-8504, Japan \\ ${ }^{b}$ Department of Electrical and Electronic Engineering, Tokyo University of Agriculture and Technology, 2-24-16 \\ Naka-cho, Koganei-shi, Tokyo, 184-8588, Japan \\ *Corresponding Author: kazuhiro.morita.fg@ hitachi-hightech.com
}

\begin{abstract}
The semiconductor inspection equipment using an electron beam in vacuum environment is required high resolution. However, it is difficult to adjust a characteristic of the XY stage working in vacuum chamber. We propose a design method for the XY stage in vacuum environment by using disturbance observer.
\end{abstract}

Keywords: vacuum, observer, feedback control.

\section{Introduction}

By the development of a smartphone and the tablet PC, the high integration of the semiconductor device advances. According to the ITRS, a design rule will become $10 \mathrm{~nm}$ in 2020. Critical dimension scanning electron beam micro-scope(CD-SEM) is used for the inspection of these semiconductor devices. It has a characteristic to inspect in a vacuum environment. Positioning precision of $2 \mathrm{~nm}$ is necessary for the sample movement stage to inspect a circuit pattern of $10 \mathrm{~nm}$ in CD-SEM.

On the other hand, cost reduction is an important theme for a developer of CD-SEM. We have to develop it has high precision while suppressing a cost. The XY stage using the air bearing is put to practical use for a semiconductor photolithography equipment ${ }^{(1)(2)}$. However, it is difficult to get the technique in a CD-SEM because there are two problems which are the cost and the technical difficulties to introduce the technique into a vacuum environment. On the other hand, since it has transformation by the heat transfer (3), it is difficult to make high precision ball-screw drive stage.
In such background, we developed a friction drive type stage using the linear motor of the direct drive. Its purpose is to manage both the cost and the precision. However, it is not easy to control the friction drive type stage because it has a dependence about the position, the pressure, and the thrust. Therefore, we decided to adopt the linear control method in this development of the stage control.

In this paper, we propose one solution technique to realize high accuracy positioning in the vacuum environment using the friction drive type stage. It is important for making an equipment with low cost.

At first, we show an issue of the friction drive type stage in the vacuum environment. Secondly, we propose a control method using the disturbance observer. Finally we show the evaluation result by the experiment.

\section{Structure of the XY Stage}

In this chapter, we clarify an issue of the XY stage by showing the structure and the characteristic that.

\subsection{Structure of a CD-SEM}

We show outline structure of the CD-SEM in Fig. 1. At first, the sample to be measured carries into the vacuum chamber. Then, the sample is loaded onto the XY stage that is in the vacuum chamber. After that, the sample changes measurement point by movement of the XY stage. The electron beam irradiated from a column is deflected by a deflection lens and is irradiated to a circuit pattern formed on a sample. The circuit pattern is inspected by using an image provided by the scanning of the electron beam. The vibration damping base is located under the vacuum 
chamber, and it controls vibration for high accuracy measurement.

\subsection{Structure of the XY Stage}

In this section, we show the structure and the characteristics of the $X Y$ stage driven in a vacuum environment. We show an outline of the XY stage in Fig. 2. The top table fixing a sample is connected to a linear motor through a guide. Therefore, the top table moves two dimensional surface by driving a linear motor. The position of the top table is measured using a laser interferometer.

\subsection{Characteristics of the XY Stage}

Though the XY stage are assembled and adjusted in the atmosphere environment, the XY stage is operated and moved in the vacuum environment of the $10^{-5} \mathrm{~Pa}$. This difference in pressure causes a change in the characteristic of the XY stage. In addition, a pressure change causes the change of friction properties. In Fig. 3, we show the position dependence properties of the XY stage. Even if the $\mathrm{XY}$ stage is driven same drive current, a characteristic of the XY stage varies according to a position.

Then, we show the thrust dependence properties of the $\mathrm{XY}$ stage in Fig. 4. We show frequency properties per drive

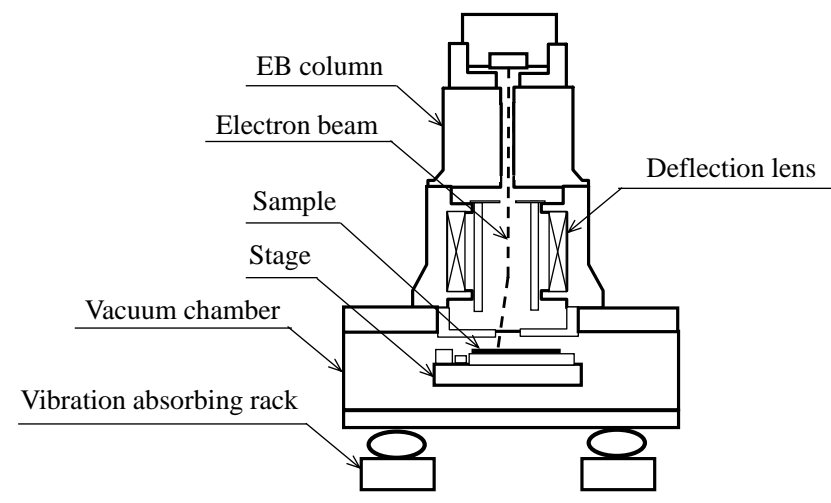

Fig. 1. Structure of a CD-SEM.

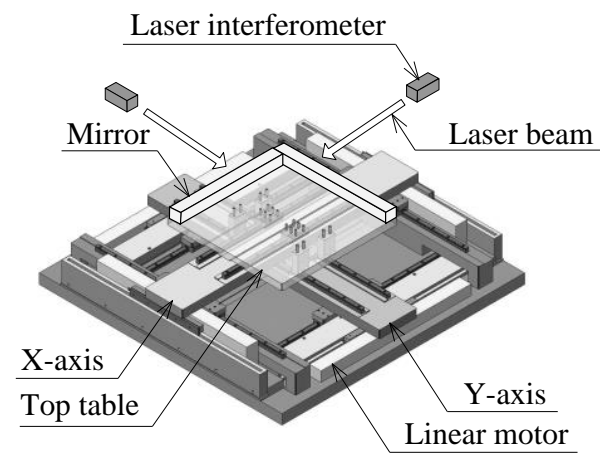

Fig. 2. Structure of XY Stage. voltage of the linear motor. We understand that characteristics are different if the drive voltage is different even if at the same position.

\subsection{Model of the XY Stage}

As we said in section 3.2, we understand that the XY stage using the guide varies its characteristic by a drive condition. In this section, we give the model of the XY stage.

At first, we give the nominal model of the XY stage. The nominal model shows a characteristic as an inertial model. In Fig. 5, we show an inertial model. It is easy to get a model of the XY stage from Fig. 5. At this time, we get Eq. (1) because the thrust $f(t) \mathrm{N}$ is proportional to driving current $i(t)$ A. $K_{t}$ is thrust constant number N/A.

$$
K_{t} i(t)=m \ddot{x}(t)+c \dot{x}(t)
$$

Then, we think about a model when a thrust is small. The XY stage indicates elastic displacement when the XY stage moves nanometer order displacement. In Fig. 6, we show elastic displacement model. As Eq. (2), we can show the micro-displacement by the elastic displacement.

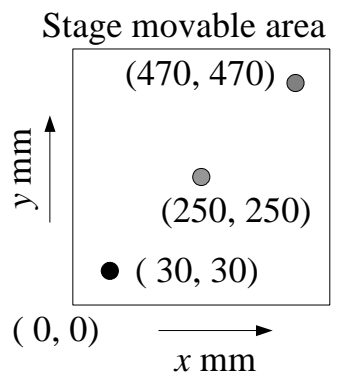

(a) Measurement points.

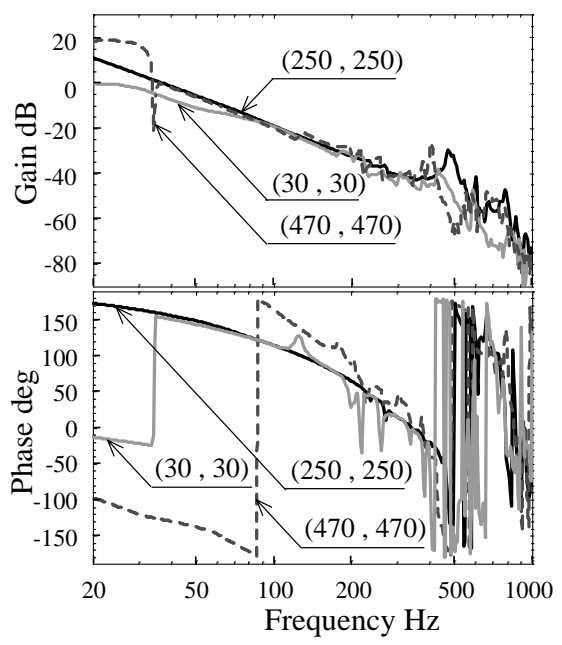

(b) Bode diagram.

Fig. 3. Indicating the Position Dependence. 


$$
K_{t} i(t)=k\left(y(t)-x_{0}(t)\right)
$$

Here, $k$ is in an elastic coefficient $\mathrm{N} / \mathrm{m}, x_{0}(t)$ is an elastic origin position $\mathrm{m}, x(t)$ is an elastic displacement $\mathrm{m}$ from $x_{0}$ is the origin position. A coefficient of viscosity $c$ changes at the same time then in $(c+\Delta c)$, too. According to Figs. 5 and 6 , when the amplitude of the input signal is large, the XY

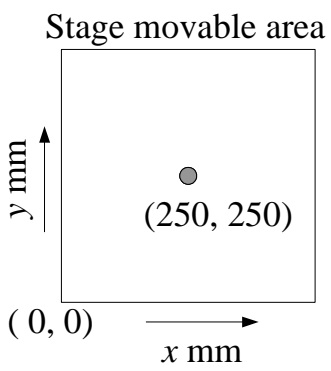

(a) Measurement points

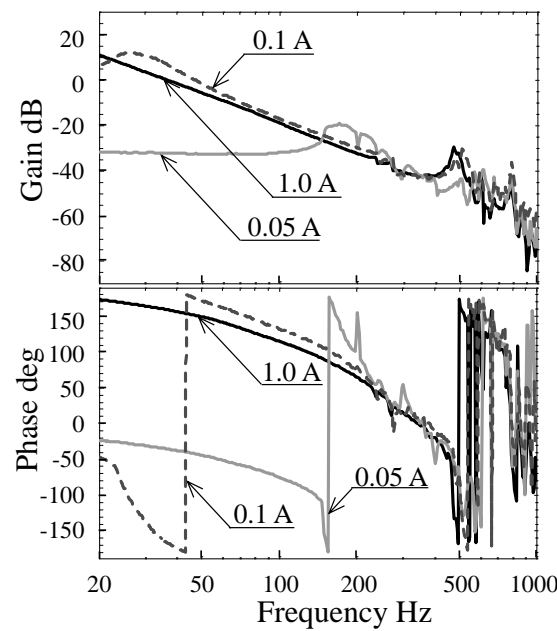

(b) Bode diagram.

Fig. 4. Indicating the Thrust Dependence.

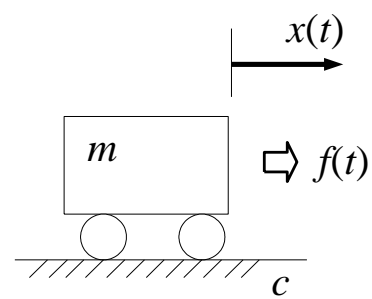

Fig. 5. Inertial Model of the XY Stage.

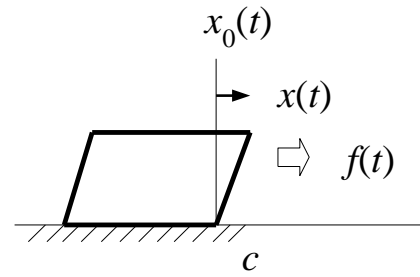

Fig. 6. Elastic Deformation Model of the XY Stage. stage shows the characteristic of the inertial model. On the other hand, the XY stage shows the characteristic as the second delay element when the amplitude becomes small. It looks like a spring was added to the inertial model. Since Fig. 4 shows a characteristic same as this model, the input-dependent characteristic of Fig. 4 accords with the concept of this elastic deformation model.

Displacement by the inertia becomes dominant when the input amplitude is large, and the elastic displacement can ignore. Then we can express the model of the XY stage is including the elastic displacement as Eq. (3).

$$
K_{t} i(t)=m \ddot{x}(t)+(c+\Delta c) \dot{x}(t)+k\left(x(t)-x_{0}(t)\right)
$$

However, in Eq. (3), it is difficult to measure the $x_{0}(t)$ is the origin position of the elastic displacement and the $\left(x(t)-x_{0}(t)\right)$ is an elastic displacement. Therefore we thought the model is assumed that the elastic displacement is disturbance. In addition, we decided to consider that the frictional force by a change of the $\Delta c$ is as disturbance. When we define the $f_{d}(t) \mathrm{N}$ for a disturbance force, the model of the XY stage can change into Eq. (4).

$$
\begin{aligned}
& K_{t} \dot{i}(t)=m \ddot{x}(t)+c \dot{x}(t)+f_{d}(t) \\
& f_{d}(t)=\Delta c \dot{x}(t)+k\left(x(t)-x_{0}(t)\right)
\end{aligned}
$$

Furthermore, we decided to consider constant disturbance of Eq. (5) as disturbance force.

$$
\dot{f}_{d}(t)=0
$$

When we summarize the above, we can express the model of the XY stage as block diagram of Fig. 7. Also, in table 1, we show the physical constant of the XY stage model that is getting from machine structure and frequency properties.

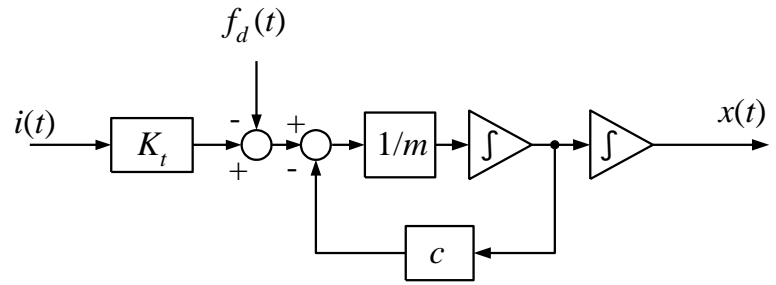

Fig. 7. Block Diagram of the XY Stage.

Table 1. Physical Parameters.

\begin{tabular}{ll|l|l}
\hline \multicolumn{1}{c|}{ Parameter } & \multicolumn{1}{c|}{ Value } & \multicolumn{1}{c}{ Unit } \\
\hline Mass of XY stage & $m$ & 46.8 & $\mathrm{~kg}$ \\
Damping coefficient & $c$ & 5.0 & $\mathrm{Ns} / \mathrm{m}$ \\
Thrust constant & $K_{t}$ & 242.0 & $\mathrm{~N} / \mathrm{A}$ \\
\hline
\end{tabular}


If we can estimate the disturbance force $f_{d}(t)$ is based on Fig. 8 and we can cancel it, the XY stage can improve the characteristic of it because the control properties to be able to treat it as linear model. After chapter 3, we give a technique of an estimate and its canceling of a disturbance force $f_{d}(t)$.

\section{Design of the Disturbance Observer and Controller}

In this chapter, we give the observer estimating disturbance $f_{d}(t)$ from the result of chapter 3 . Then we give the controller with canceling the disturbance force.

\subsection{Derivation of the State Equation}

At first, we derive the state equation of the XY stage model. We get a state equation of Eq. (6) from Eqs. (4) and (5).

$$
\begin{aligned}
& \dot{\boldsymbol{X}}(t)=\boldsymbol{A} \boldsymbol{X}(t)+\boldsymbol{B} i(t) \\
& x(t)=\boldsymbol{C} \boldsymbol{X}(t)
\end{aligned}
$$

Here, $\boldsymbol{A}, \boldsymbol{B}, \boldsymbol{C}, \boldsymbol{X}(t)$ are as follows.

$$
\begin{aligned}
& \boldsymbol{A}=\left[\begin{array}{ccc}
-\frac{c}{m} & 0 & -\frac{1}{m} \\
1 & 0 & 0 \\
0 & 0 & 0
\end{array}\right], B^{\mathrm{T}}=\left[\begin{array}{ccc}
\frac{K_{t}}{m} & 0 & 0
\end{array}\right], \boldsymbol{C}=\left[\begin{array}{lll}
0 & 1 & 0
\end{array}\right], \\
& \boldsymbol{X}^{\mathrm{T}}(t)=\left[\begin{array}{lll}
\dot{x}(t) & x(t) & f_{d}(t)
\end{array}\right]
\end{aligned}
$$

Since a rank of the observability matrix from Eq. (6) is 3 , this system is the observable.

\subsection{Design of the Disturbance Observer}

At first, since dimension of the reduced order estimator (After that we call it ROE) is smaller more than the full order estimator (After that we call it FOE), we designed the disturbance observer using a method of the ROE that estimates a disturbance $f_{d}(t)$. Since the ROE has less

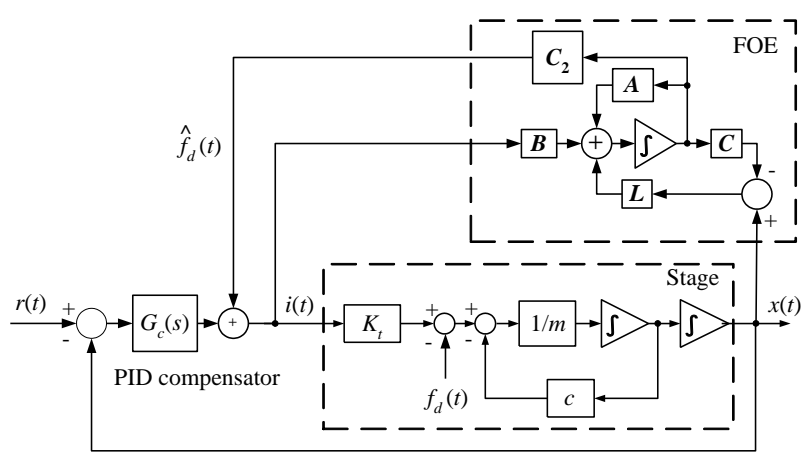

Fig. 8. Block Diagram of the XY Stage with the FOE. computational complexity than the FOE, it is easy to implement the ROE. However, the ROE was difficult to estimate it stably, and it was not able to estimate $f_{d}(t)$ whole area of the XY stage. In addition, the pole of the ROE convergence was slower than the FOE. Therefore, we decided to adopt the FOE.

Based on the FOE of Luenberger ${ }^{(4)}$, we estimate $\boldsymbol{X}(t)$ that is including disturbance $f_{d}(t)$. We think about the feedback control model of the output error to get $\hat{\boldsymbol{X}}^{\mathrm{T}}(t)=\left[\begin{array}{lll}\hat{\dot{x}}(t) & \hat{x}(t) & \hat{f}_{d}(t)\end{array}\right]$ that is the estimated $\boldsymbol{X}(t)$.

That model is shown in Eq. (7). Here, $\boldsymbol{L}$ is a feedback gain matrix.

$$
\dot{\hat{\boldsymbol{X}}}(t)=(\boldsymbol{A}-\boldsymbol{L C}) \hat{\boldsymbol{X}}(t)+\boldsymbol{B} i(t)+\boldsymbol{L} x(t)
$$

We used the LQ control method to get matrix $\boldsymbol{L}$ which is able to keep robustness. We considered a quadratic form evaluation function of Eq. (8) as evaluation function of the LQ control method. In Eq. (8), $\boldsymbol{Q}$ is a positive semidefinite matrix and $R$ is a positive number. Then, the feedback gain $\boldsymbol{L}$ to minimize evaluation function $J$ is $\boldsymbol{L}=R^{-1} \boldsymbol{C}^{\mathrm{T}} \boldsymbol{P}$. Here, $\boldsymbol{P}$ is the unique positive definite matrix satisfying the Riccati equation.

$$
J=\int_{0}^{\infty}\left(\boldsymbol{X}^{\mathrm{T}} \boldsymbol{Q} \boldsymbol{X}+R i^{2}\right) d t
$$

In this paper, we decided $\boldsymbol{Q}, R, \boldsymbol{L}$ by simulation as follows.

$$
\boldsymbol{Q}=\left(\begin{array}{ccc}
1 & 0 & 0 \\
0 & 1 & 0 \\
0 & 0 & 10^{27}
\end{array}\right), \quad R=1, \quad \boldsymbol{L}=\left(\begin{array}{c}
2.543769 \\
1.789624 \times 10^{4} \\
-3.035787 \times 10^{9}
\end{array}\right)
$$

\subsection{Design of the Feedback Controller}

In section 3.2, we designed the observer to estimate disturbance. In this section we give the feedback controller to cancel disturbance by using the estimated disturbance $\hat{f}_{d}(t)$.We get $\hat{f}_{d}(t)$ from Eq. (9). In Eq. (9), it is $\boldsymbol{C}_{2}=\left[\begin{array}{lll}0 & 0 & 1\end{array}\right]$.

$$
\hat{f}_{d}(t)=C_{2} \hat{X}(t)
$$

In Fig. 8, we show a block diagram of the FOE that is including the feedback of $f_{d}(t)$. We can get linearity by a feedback of the $\hat{f}_{d}(t)$. As a result, we can control the XY stage precisely with a simple PID compensator.

\section{Result of an Experiment}

Based on the result of chapter 3, we conducted a confirmation experiment to control the XY stage in a vacuum environment. In this section, we show result of an experiment. 


\subsection{Frequency Characteristic}

In this section, we show suppressive effect of the characteristics fluctuation by comparison of frequency characteristics. At first, we measured a frequency characteristics to confirm the suppressive effect of the position dependance. In Fig. 9, we show an open-loop Bode diagram of the XY stage on each position. We can see suppressing effect of a fluctuation about a gain and a phase. Natural frequency fluctuates from 330 to $450 \mathrm{~Hz}$, but it does not have issues for positioning control because it is in a high frequency and a high damping. Then, we measured frequency characteristics that depends on the amplitude of the input. In Fig. 10, we show a Bode diagram at the XY stage position $(250 \mathrm{~mm}, 250 \mathrm{~mm})$. The fluctuation of a characteristic of a gain and a phase is reduced by the effect
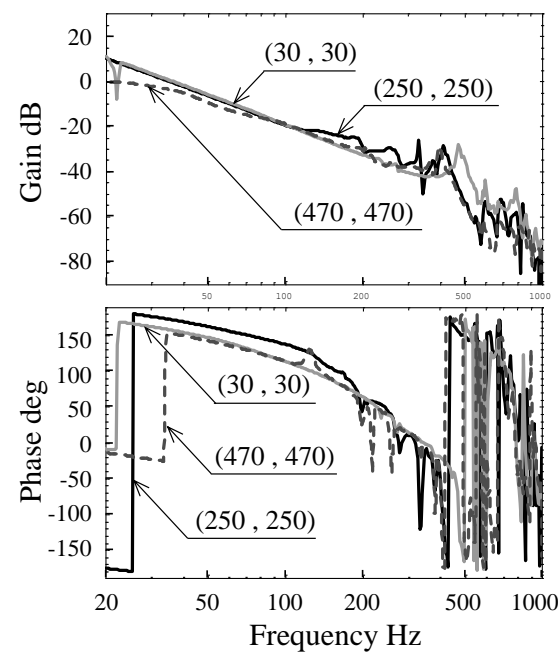

Fig. 9. Frequency Characteristics Depending on Changing Position.

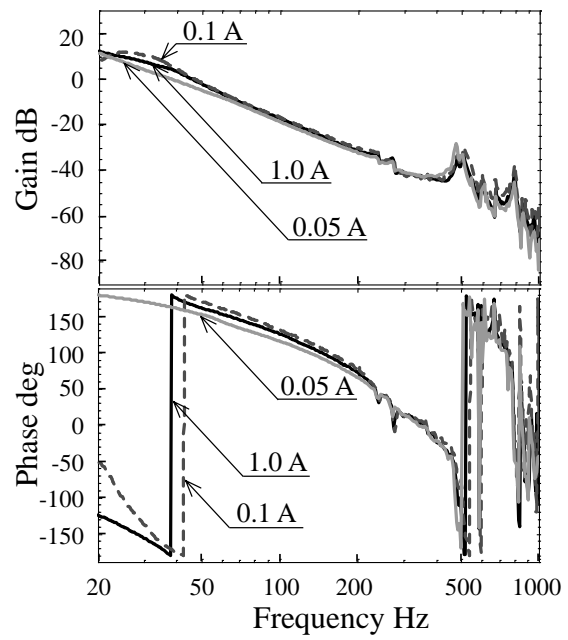

Fig. 10. Frequency Characteristics Depending on Input Current. of feedback an estimated disturbance.

\subsection{Time Response}

In Fig. 11, we show the control result by the difference of the XY stage position. It can improve positioning performance by feedback disturbance in all positions. Positioning precision improved to $10 \mathrm{~nm}$ from $30 \mathrm{~nm}$ by addition of the FOE. It meets specifications. In Fig. 12, we show the step response in vacuum environment. In table 2, we show a condition for stage movement. According to Fig. 12 , a settling time and an overshoot value of the XY stage are improved by using the FOE.

\section{Conclusions}

We showed that even if there is the $\mathrm{XY}$ stage in the vacuum environment, the feedback control by using the disturbance observer was effective. It can build a stable positioning control system under the vacuum environment

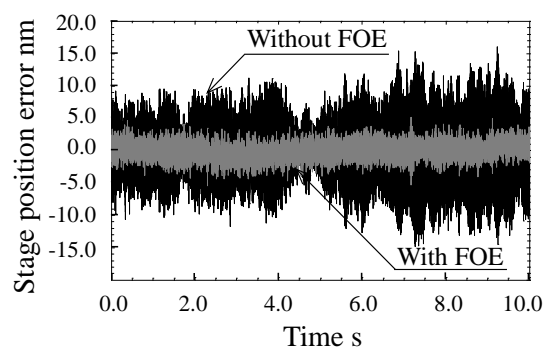

(a) $(30 \mathrm{~mm}, 30 \mathrm{~mm})$

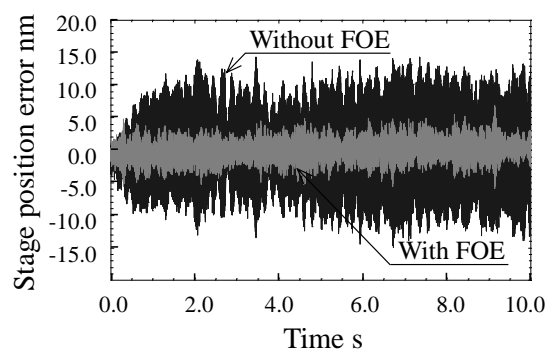

(b) $(250 \mathrm{~mm}, 250 \mathrm{~mm})$

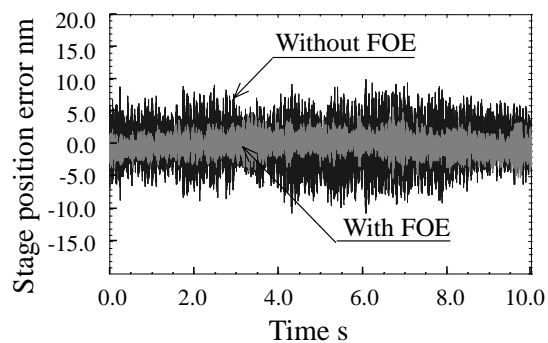

(b) $(430 \mathrm{~mm}, 430 \mathrm{~mm})$

Fig. 11. Time Response Depending on Stage Position. 
which is difficult of precise adjustment. Furthermore, precise positioning was realized by the simple control system using the PID compensator under the vacuum environment because a characteristic of the disturbance observer was stable.

\section{References}

(1) Hiroo Kinohita, Munenori Kanai, Kimiyoshi Deguchi, and Tadao Saito : "Air Bearing Guided High Speed XY Stage", Journal of the Japan Society for Precision Engineering, Vol. 52, No. 10, pp. 1713-1718, 1986

(2) Yoshiyuki Tomita, Eiji Kojima, Shunichi Kawachi, Yasushi Koyanagawa, and Seiji Ootsuka : "Development and Applications of Sumitomo Precision Stage Technologies for FPD Process", Journal of the Japan Society for Precision Engineering, Vol. 78, No. 2, pp. 117-121, 2012

(3) Kazuhiro Yokoyama, Mizuki Saito, Hiraku Torinoumi,

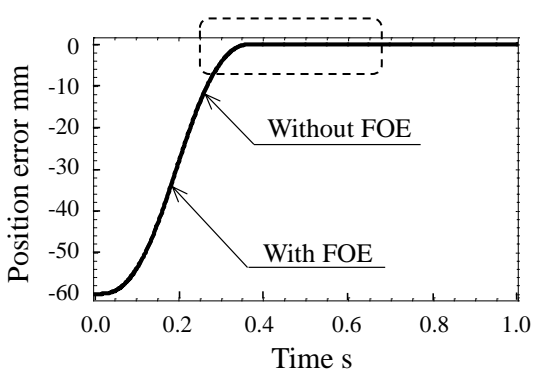

(a) Comparison of position error at step movement by having the FOE or not.

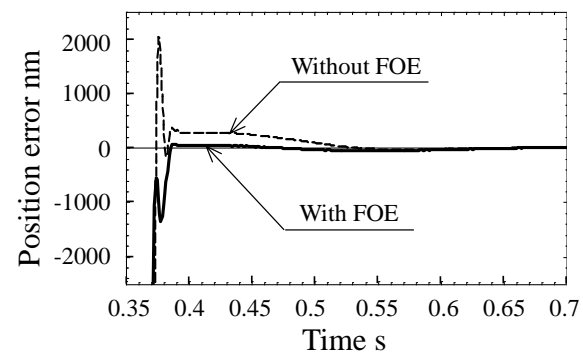

(b) Close-up of position error at step movement by having the FOE or not.

Fig. 12. Step Response in Vacuum. Environment.

Table 2. Condition of a Step Movement.

\begin{tabular}{l|l|l}
\hline \multicolumn{1}{c|}{ Condition } & \multicolumn{1}{|c}{ Value } & Unit \\
\hline Moving distance & 60 & $\mathrm{~mm}$ \\
Max. acceleration & 1.96 & $\mathrm{~m} / \mathrm{s}^{2}$ \\
Max. velocity & 0.3 & $\mathrm{~m} / \mathrm{s}$ \\
Pressure in a chamber & $2.31 \times 10^{-3}$ & $\mathrm{~Pa}$ \\
\hline
\end{tabular}

Toshimichi Moriwaki, and Masato Aketagawa : "Improvement of Positioning Accuracy by Real-Time Compensation of Thermal Elongation of Ball-screw( $1^{\text {st }}$ Report) - Principle of the Compensation and Influence of Driving Condition -", Journal of the Japan Society for Precision Engineering, Vol. 74, No. 9, pp. 931-936, 2008

(4) D.Roy Choudhury : "Modern Control Engineering", PHI Learning, pp. 650-658, 2006 